\title{
The Role of Simulation in Designing for Universal Access
}

\author{
Simeon Keates ${ }^{1}$, Peter Olaf Looms ${ }^{2}$ \\ ${ }^{1}$ School of Engineering, University of Greenwich, Medway, Kent, UK \\ s.keates@gre.ac.uk \\ ${ }^{2}$ Technicsl University of Denmark, Lyngby, Denmark \\ polooms@gmail.com
}

\begin{abstract}
It is known that the adoption of user-centred design processes can lead to more universally accessible products and services. However, the most frequently cited approach to user-centred design, i.e. participatory design, can be both problematic and expensive to implement., particularly over the difficulty of finding and recruiting suitable participants. Simulation aids offer a potentially cost-effective replacement or complement to participatory design. This paper examines a number of the issues associated with the use of simulation aids when designing for Universal Access. It concludes that simulation aids can play an effective role, but need to be used with due consideration over what insights they provide.
\end{abstract}

Keywords. User-centred design, universal access, simulation, impairments

\section{Introduction}

What makes a successful product? This is a question that designers and design commissioners ponder regularly. In many cases, the question can be answered by reference to a property or attribute of the design, such as, for example, the fastest, the cheapest or the most reliable. Such attributes can be measured and quantified by direct empirical analysis.

However, when designing for use by people, the attributes commonly cited suddenly become decidedly more woolly and imprecise. Words and phrases such as "user friendliness," "intuitiveness" and "user experience" are used. While these phrases make some sense at a surface level, once you start to examine them more closely, they become increasingly unsatisfactory as design requirements. For example, there is no universally accepted definition of what constitutes a user-friendly or intuitive design. Similarly, and as a direct consequence of the difficulty in defining them, there is no clear approach to how to measure them.

A review of the literature shows many different approaches and no single, uniform, "best practice" approach to designing for people. The closest to a conformity of opinion in this area is that a user-centred design approach is the most reliable option for generating a usable, user-friendly, intuitive, etc., design.

In an attempt to minimise the variability in the design requirements and specifications, designers will typically try to reduce the users to a simple homogeneous representative. As Cooper warns [7], unless they are presented with a very specific view of 
who the users are, the designers will often substitute themselves as the target users. This is a seductive assumption to make. After all, designers are people and the users are people, so who is to say that the designers are not suitably representative of the target users?

Of course, taking a quick step back to view the problem and the problem with that assumption becomes clear. For example, not all users, and indeed very few users, will have the insights into the operation of the product or system that the designer will have. Consequently, the designers will be power users, whereas most target users will not. A single designer is also, by definition, homogenous. By designing for him or herself, the designer is making the assumption that all users will share his or her knowledge and also his or her physical attributes and functional capabilities. This raises the question of how valid is that assumption? The answer is often that it is not valid. Furthermore, if the assumption is not valid, how can the designers be assisted in designing products or services that better meet the needs of the wider population?

Those who endorse user-centred design typically focus on the direct participation of users in the scoping, development or subsequent optimisation of their products. Others make use of proxies for target users in the design process by using personas. In this paper, however, we will examine the role that simulation can play in assisting designers design for the widest possible range of users.

\section{Designing for Universal Access}

For many people, a disabled person is typified by either a young man in a wheelchair or an older blind man walking with either a white cane or a guide dog. However, these are both anachronistic stereotypes that do not reflect the true variety or prevalence of functional impairments across the whole population. For designers, it is functional impairment - i.e. a limitation in someone's capabilities - that is important. Disability is a consequence of a person's functional impairments preventing them from interacting with a product or service successfully within a given context [11]. If the product or service is designed to be sufficiently robust to support or accommodate a wide enough range of functional impairments or limitations, then no disability or handicap should be experienced by that person.

The most common approach to designing for Universal Access is to adopt a participatory design approach, i.e. recruiting users into the design. This is the basis taken for the Design Business Association (DBA) Inclusive Design Challenges, organised in conjunction with the Helen Hamlyn Centre [4]. DBA member consultancies from all design disciplines are set a design challenge to create a mainstream product or service that can be enjoyed equally by users of all abilities. The teams work with the Helen Hamlyn Centre, disabled users and other experts to ensure that all aspects of inclusivity are considered throughout the Challenge. Prizes are awarded at the end of each Challenge.

While participatory design approaches do, in general, lead to more inclusive designs when appropriate users have been selected, it can be difficult to find and recruit the 
users. Even when users have been found and recruited successfully, the costs of organizing consultation sessions can be significant. Furthermore, there are questions over the representative nature of such a design approach. How, for example, does the design team choose which participants to select and include? Fundamentally, how can the design team ensure that the outcome of the participatory design process is a product or service that is accessible by many potential users and not simply those who are sufficiently similar to the members of the participatory design team? Many accessibility audit panels typically consist of a very small number of users. How does the design team ensure that the capabilities of those users reflect the wider population? We can go further, can such panels ever truly represent the wider population?

These are difficult questions to answer. To begin to do so, it is necessary to understand the prevalence of functional impairments.

\section{Prevalence of functional impairments}

Functional impairments are surprisingly prevalent across the population. For example, in 1996/7 it was estimated that $17.8 \%$ of the population of Great Britain (i.e., England, Wales and Scotland) had at least one functional impairment [9]. These impairments could be further broken down into:

- $14.7 \%$ having a motor impairment, such as locomotion, dexterity, reach and stretch, strength)

- $5.7 \%$ having a cognitive impairment, such as difficulty with memory, recall, recognition, understanding and communication

- $8.7 \%$ having a sensory impairment, such as vision or hearing

It can be seen that $14.7 \%+5.7 \%+8.7 \%>17.8 \%$. This inequality arises because approximately $8.8 \%$ of the population has more than one class of functional impairment, e.g., a motor and a cognitive impairment. $2.5 \%$ of the population has all three classes of impairment, i.e., motor, cognitive and sensory.

This overall prevalence pattern is believed to be typical of the developed world. The US Census Bureau's 1999-2004 American Community Survey [1] asked respondents if they had any kind of disability, defined here as "a long-lasting sensory, physical, mental or emotional condition," a definition that fits well with that in Article 1 of the UN Convention on the Rights of Persons with Disabilities [18]. The data collected were as follows:

- $16.0 \%$ reported any type of disability (cf. $17.8 \%$ from the British survey)

- $4.7 \%$ reported a sensory disability (cf. $8.7 \%$ for a sensory impairment)

- $10.6 \%$ reported a physical disability (cf. $14.7 \%$ for a motor impairment)

- $5.2 \%$ reported a mental disability (cf. 5.7\% for a cognitive impairment)

- $3.1 \%$ reported a self-care disability (no direct comparison available)

- $4.9 \%$ reported a go-outside-home disability (no direct comparison available)

- $5.6 \%$ reported an employment disability (no direct comparison available) 
Again, it can be seen that multiple impairments/disabilities are common. From the same survey:

- $6.7 \%$ reported one type of disability (cf. $5.9 \%$ )

- $7.6 \%$ reported two or more types of disability (cf. 8.8\%)

Some of the differences in prevalence will arise from the different definitions used for each survey. A second issue is that of self-reporting: some individuals may well have an impairment of which they are not aware or that they do not consider to be serious. A study from 2012 of the intelligibility of television audio from the public service broadcaster DR in Denmark, discussed in [16], suggests that the prevalence of hearing impairments may be markedly higher than was shown in the studies by Grundy and American Community. However, if we restrict ourselves to the methodologies used in these two studies, the overall pattern is sufficiently similar for it to be assumed that no less than 1 in 6 adults in a developed country will have a functional impairment and approximately 1 in 12 will have two or more classes of impairment types.

It is also worth noting that prevalence of disability typically increases with age [5]. Although older adults are increasingly healthy compared with their predecessors, the ageing process is still accompanied by an overall decrease in functional capabilities. Typically several capabilities will degrade over time and this leads to the widespread prevalence of multiple impairment classes. Someone who is older could easily have arthritis, cataracts and be a little hard of hearing, for example.

One very useful source of information is quantified data about the extent of exclusion caused by each item on the list of issues to be fixed. Effectively, each source of exclusion can be ranked by how many people are excluded by that particular problem, e.g. how many people cannot see that label and how many people cannot hear that beeper. Exclusion calculators, based on the data collected for the UK 1996/7 Disability FollowUp Survey are available at:

- http://www.inclusivedesigntoolkit.com/

- http://www.eng.cam.ac.uk/inclusivedesign/

These calculators allow designers to specify the capability demands placed on the users by each stage of the interaction with the product. The calculators then display how many adults within the population of Great Britain cannot meet those demands, and thus would be excluded from using the product. Worked examples of how to use the calculators have been provided by Keates and Clarkson [6, 10].

These data can be used to inform user-centred design processes when designing for Universal Access.

\section{$4 \quad$ Implementing user-centred design}

The principal methods most commonly associated with user-centred design are participatory design, co-operative design and contextual design. Participatory design and cooperative design are largely very similar, with the principal difference being that participatory design is more widely used in Europe and co-operative design in the US. 
However, they both focus on the role of users as equal members within the design team. Contextual design arose from ethnographic methods, building up a better understanding of who the users are and the wider context of use of the product to be designed [2].

Where a wholly new design for a product or service is required, any of these usercentred approaches are valid and, if implemented correctly with an appropriate choice of users, should yield an accessible and inclusive design. However, these methods are expensive and time-consuming to adopt and so organisations are typically looking for cheaper and faster options for achieving similar outcomes. Such options can include:

- Empathy. Empathising with the users is a very cheap method for implementing user-centred design. Put simply, the designers try to picture themselves as the users and cognitively walk through the process of using a product or service from that users perspective. Indeed, contextual design could be considered a highly developed variant of this. The effectiveness of this approach can be enhanced through videos, photographs, multimedia stories, etc. of the users. There are numerous websites available that offer invaluable information to support this approach, such as the Inclusive Design Toolkit. The principal disadvantage to this approach is how it is calibrated. In other words, how do the designers ensure that what they think is the case, really is the case? That calibration needs to happen via supplementary or complementary methods, such as user evaluation / observation sessions.

- User evaluation or user observation sessions. Rather than recruiting users for the entirety of the design process, organisations may opt to recruit them for intensive sessions where the users interact with the product or service. The observations are then used in the re-designing of features that were problematic for the users. However, further iterations of user sessions are then required to ensure that the re-designed product is genuinely an improvement. One common approach is a frequent cycle of user evaluations at defined gateways in the design and development process. However, there is also a balance to be reached regarding the frequency of such sessions. Too frequent and the process becomes expensive and time-consuming. Too infrequent and the design being evaluated may have acquired more inaccessible features as the design will have progressed substantially in the intervening time period. The user sessions are required to keep the design "on track."

- Simulation aids. The physical aspects of particular impairments or capability limitations can be simulated through the use of aids, such as thick gloves (loss of feeling), ear defenders (hearing impairment), fuzzy screen shots (minor visual impairments) and a blindfold (blindness). Such aids can help identify many basic accessibility issues quite quickly. However, it is possible to read too much into simulation and both designers and researchers have been known to regard simulation as reproducing the entire experience of what it is to have a particular impairment instead of recognising that only one aspect of the impairment is actually being simulated.

The remainder of this paper will focus on the potential role of simulation and simulation aids in designing for Universal Access. 


\section{$5 \quad$ Approaches to simulation}

There are two principal approaches to the role of simulation and simulation aids:

- Formative simulation. It is possible to use simulation as a formative component during the creative stages of design, where concepts, prototypes and solutions are being developed. Formative simulation may involve the prototype product or the underlying system that gives rise to the prototype (for example a TV programme with subtitles/closed captions or the end-to-end system for the production and distribution of a programme with subtitles/closed captions). If a simulation aid affords a sufficiently high fidelity insight into the difficulties faced by potential users, designers can benefit from this to proactively design solutions that accommodate the needs of those users. Effectively, the simulation aids help shape, or form, the designers' understanding as they generate new solutions.

- Summative simulation. The other approach to using simulation aids is when reviewing designs to evaluate the accessibility of a design once it has been created. Designers can use the simulation aids to retrospectively amend and optimise a design as part of an iterative design process. It could be argued that this is a reactive, rather than proactive, use of simulation aids. EIII, a major research project looking into 'crowd-sourcing' to get users of Internet resources to assess the accessibility of (public) websites uses this approach [8].

Many simulation aids can be used as part of either of the above approaches.

While it is generally appreciated that proactive design approaches are more effective and cost-effective than reactive ones, especially in the area of Universal Access, there is a fundamental question about how much of an insight into a user's true condition can be gained through a simulation aid. For example, does closing your eyes really help in understanding what it is like to be blind? It could be argued, on a surface level, that indeed it does. Certainly, closing your eyes does bring home the immediacy of dangers, such as nearby hazards. However, it can equally be argued that this level of understanding is largely symptomatic, not causal. In other words, you can simulate the consequence of being blind, but not really the full experience of it as a long-term condition.

This distinction is important. Anyone who has spent time working with or observing people with severe impairments will usually notice that such people often develop unexpected techniques for interacting with everyday products and technologies. These are the so-called "coping strategies." Users develop these strategies to take advantage of their comparatively non-impaired capabilities. For example, there are cases of people who cannot use their hands developing the skill of holding a cup with their feet. It is questionable whether a temporary simulation of one aspect of someone's functional impairment would necessarily lead to a designer attempting this particular feat.

Consequently, it could be argued that simulation aids serve a much better purpose as reactive design tools, where simply closing your eyes will help evaluate whether the functionality of a product can be accessed by someone who is blind. However, even this approach is not without its limitations. The principal drawback is that this approach is based on the notion that the designers will only really learn whether a product is 
basically accessible, i.e. that a user can gain basic access to its functions. It does not guarantee that the product will be usable or accessible in an acceptable amount of time. It also, almost certainly, does not allow for whether a user's coping strategies may help overcome some of the difficulties encountered by adopting a different approach, based on potentially years of experience, that a user may take that a designer may not predict.

However, to truly evaluate the usefulness and role of simulation, it is worth examining a few examples of it in use.

\section{Simulation in practice}

There are many different types of simulation aid ranging from very simple simulation of one type of functional impairment, such as closing your eyes or covering your ears, to highly sophisticated simulators of multiple impairment types.

\subsection{Simulating colour blindness}

About $8 \%$ of the western male population is colour blind. Vischeck [19] is a freely available online tool that can simulate the effects of Deuteranopia (red/green colour deficit), Protanopia (red/green colour deficit) and Tritanopia (blue/yellow colour deficit) by applying different colour filters to screenshots or web sites. The tool is straightforward to use and the results are believed to be realistic.

However, when used by postgraduate students in a Masters course at the IT University of Copenhagen, it was found that the output was sometimes difficult for the students to interpret correctly. A small number of groups tried to adjust the colour schemes of their sites to still look visually appealing to themselves, while not appreciating that their colour preferences (with unimpaired colour vision) were not the same as for someone with a colour vision impairment. Most students, though, used Vischeck to look for insufficient colour contrast for each of the three colour vision impairment types, which was usually more successful and is the generally accepted correct use of the tool.

\subsection{Simulating computer access difficulties}

There are several possible methods for simulating the potential difficulties experienced when access computer and IT systems. For example, simulating the effects of vision impairment can be achieved at some level by switching off the monitor or computer display and relying upon a screen reader, such as JAWS, to provide information about the display. This is a common accessibility evaluation technique and provides good insight into potential problems, especially when combined with the use of the W3C HTML validation tools.

Similarly, simulating the effects of hearing impairment is straightforward. All that needs to be done is to mute the sound output from the computer system.

Simulating motor impairment is more difficult. While work has been done on understanding, quantifying and modelling the movements of users with motor impairments 
(e.g. [13, 14]), there is a question over the most appropriate method of using such quantified and calibrated models. For example, consider modelling the effects of spasms on user cursor control. A spasm can occur at any point in an interaction, but is most commonly experienced close to a target. A probabilistic model for the onset of a spasm can be developed based on sufficient empirical data. Similarly, it is straightforward to perform a frequency analysis of essential tremor to develop a mathematical model of the resultant cursor movements.

However, there is then the issue of what to do with such models. It is also straightforward to apply them to the cursor movement of an able-bodied user and thus introduce artificial noise into the cursor behaviour. The problem, though, is that such noise would only be experienced visually by the user and the kinaesthetic feedback loop between the user's eyes and arm/hand movements would be broken, i.e. the user would see the noise, but not feel it. It is debatable how useful such simulation would be in terms of establishing how easy an on-screen target would be to hit using such an approach. One solution is the use of haptic force feedback, so the user can feel the noise being generated, thus re-establishing the kinaesthetic feedback paths [15].

In the absence of haptic feedback, the most appropriate solution here would be to run the model as a full probabilistic simulation of the interaction, i.e. one where the computer controls the cursor and the noise and simply reports back the expected time to complete the task [3].

\subsection{Simulating ageing - the "Third Age Suit"}

Ford cars have traditionally been at or near the top of car sales in the UK for many years. As the venerable Ford Escort approached the end of its manufacturing lifespan, Ford began to develop a replacement model, the Ford Focus.

Unlike any car built by previously by Ford, the designers of the Focus were encouraged to design for the needs of older. Ford even went so far as to develop a novel method of simulating the effects of old age using what became known as the 'Third Age Suit' in conjunction with Loughborough University [10].

The suit was designed to simulate the equivalent of another 30 years of ageing to the wearer. This ageing effect was achieved by joint stiffeners in the neck, back, stomach and knees to simulate the reduced flexion from conditions such as arthritis. The suit added both weight and bulk around the torso to replicate both the change in body shape and the difficulty in getting into and out of cars often associated with ageing. Visual impairments, such as cataracts, are also simulated through the use of spectacles with different lenses where each lens type was marked with different patterns or colours.

Designers were encouraged to wear the suit to increase their empathy with older users by letting them experience some of the difficulties faced by older drivers. As a result of their use of the suit, the Focus offers many innovative features. For example, it has the most headroom of any cars in its class. The front door is wider and higher than in the Escort and the seats are higher. This combination of door size and seat height makes it significantly easier to get in and out of the Focus. The dashboard controls are larger than those of its predecessor and were designed to be easier to locate, grab and operate. 
All of the features developed to make the car easier to operate and drive for older adults have not adversely affected the enjoyment of the Focus for younger drivers. Indeed, many of the features introduced are of benefit to all drivers. For example, the easier access to the car is good for parents with small children. Larger, easy to use controls are good for everyone.

\subsection{Simulating cognitive impairments}

One area of simulation that is known to be problematic is that of simulating cognitive impairments. While those with visual and hearing impairments have vocal and persuasive advocates, those with cognitive impairments rarely have the same organisational support. Public awareness of the issues is lower as a result.

One category of cognitive impairments includes aphasias. Olesen and Slynge [17] report on their work in the development of rehabilitation software for people with aphasias and the need to recognise the multiplicity of manifestations this impairment may have.

Other cognitive impairments can be simulated temporarily through chemical intervention, e.g. alcohol. While possible, this is ethically dubious, to say the least. Forms of distraction or deliberate cognitive overloading of an able-bodied user may produce similar effects to lack of concentration or some memory impairments. Again, though, this is ethically questionable since it usually involves stressing the user to induce such symptoms. Arguably the safest option for designing for cognitive impairments is to follow recommended design guidelines, such as in [12].

\section{$7 \quad$ Conclusion}

Simulation aids clearly have a valuable role to play when designing for Universal Access. However, their strengths and weaknesses need to be understood by designers attempting to use them to inform the design process, whether as proactive or reactive design tools. Designers, or designer evaluators, need to understand what the simulation aid is intended to simulate. They also need to be aware that it is highly unlikely that such aids will provide a full, high-fidelity understanding of what it is to be functionally impaired as a long-term condition. As such, simulation aids need to be handled with caution. However, when they are used correctly they are capable of assisting designers in creating more universally accessible products, as clearly demonstrated in the examples given in this paper.

\section{References}

1. ACSO (2007) American Community Survey 2004 subject definitions. American Community Survey Office, US Census Bureau

2. Beyer, H. and Holtzblatt, K. (1998). Contextual Design: Defining customer-centered systems. Morgan Kaufmann, San Francisco, CA 
3. Biswas, P., Robinson, P. (2008) Automatic evaluation of assistive interfaces. Proceedings of the 13th ACM International Conference on Intelligent User Interfaces. 247-256

4. Cassim, J. (2004). Cross-market product and service innovation - the DBA Challenge example. In: Keates et al. (Eds.) Designing a more inclusive world (pp.11-19). London, UK: Springer-Verlag.

5. Christensen, K., Doblhammer, G., Rau, R., Vaupel, J.W. (2009) Ageing populations: The challenges ahead. Lancet. 374(9696), pp. 1196-1208

6. Clarkson, P.J., Keates, S. (2002) Quantifying design exclusion. In: Keates, Clarkson, Langdon, Robinson (eds.) Universal Access and Assistive Technology, (pp 23-32) London, UK: Springer-Verlag

7. Cooper, A. (1999). The inmates are running the asylum. Indianapolis: SAMS Publishing.

8. EIII (2014). European Internet Inclusion Initiative. http://eiii.eu/ (acc. 2 February, 2014)

9. Grundy, E., Ahlburg, D., Ali, M., Breeze, E., and Sloggett, A. (1999) Disability in Great Britain: results from the 1996/7 disability follow-up to the Family Resources Survey. Huddersfield, UK: Charlesworth Group.

10. Keates, S., and Clarkson, P.J. (2003) Countering design exclusion: An introduction to inclusive design. London, UK: Springer-Verlag

11. Keates, S. (2007) Designing for accessibility: A business guide to countering design exclusion. Mahwah, NJ: Lawrence Erlbaum Associates / CRC Press

12. Keates, S., Adams. R., Bodine, C., Czaja, S., Gordon, W., Gregor, P., Hacker, E., Hanson, V., Kemp, J., Laff, M., Lewis, C., Pieper, M., Richards, J., Rose, D., Savidis, A., Schultz, G., Snayd, P., Trewin, S., Varker, P. (2007) Cognitive and learning difficulties and how they affect access to IT systems. Int J on Universal Access in the Information Society, Springer, 5(4), 329-339

13. Keates, S., Langdon, P., Clarkson, P.J., Robinson, P. (2002) User models and user physical capability. User Modeling and User-Adapted Interaction (UMUAI), Wolters Kluwer Publishers 12(2-3), 139-169

14. Keates, S., Hwang, F., Langdon, P., Clarkson, P.J., Robinson, P. (2002) The use of cursor measures for motion-impaired computer users. Int $\mathrm{J}$ on Universal Universal Access in the Information Society (UAIS), Springer, 2(1), November, 2002, 18-29

15. Langdon P, Hwang F, Keates S, Clarkson PJ, Robinson P (2002) Investigating haptic assistive interfaces for motion-impaired users: Force-channels and competitive attractive-basins. Proceedings of Eurohaptics 2002 Int'l Conference, Edinburgh, 122-127.

16. Looms, P. (2014) Making TV Accessible in the 21st Century. Chapter 3: Policy and Marketing Strategies for Digital Media. Edited by Yu-li Liu \& Robert G. Picard, Routledge, Abingdon, UK. - in press

17. Olesen, Rene \& Casper Slynge (2012). Sharp - Rehabilitation Software for Aphasics. Aalborg University, Department of Media Technology, Aalborg, Denmark. http://projekter.aau.dk/projekter/files/63417640/SHARPreport_full.pdf

18. UN (2006) UN Convention on the Rights of Persons with Disabilities. http://www.un.org/disabilities/convention/conventionfull.shtml (acc. 2 February, 2014)

19. Vischeck (2008) Vischeck. Available at: http://www.vischeck.com/ (accessed 2 February, 2014) 